\title{
Accelerating Convergence of Trigonometric Approximations
}

\author{
By William B. Jones and G. Hardy
}

\begin{abstract}
Lanczos has recently developed a method for accelerating the convergence of trigonometric approximations for smooth, nonperiodic functions by modifying their boundary behavior. The method is reformulated here in terms of interpolation theory and is shown to be related to the theory of Lidstone interpolation. Extensions given include a new type of modifying function and the establishment of criteria for the convergence of associated interpolation series. Applications are given for the error function and its derivative.
\end{abstract}

1. Introduction. We are concerned with the problem of accelerating the convergence of trigonometric approximations, such as truncated Fourier series and trigonometric sums for interpolation at equally spaced points. Let $f(x)$ be a real valued function of a real variable $x$ defined on the interval $[-1,1]$. If $f(x)$ is integrable on $[-1,1]$, its Fourier series is given by

$$
\frac{a_{0}}{2}+\sum_{k=1}^{\infty}\left(a_{k} \cos k \pi x+b_{k} \sin k \pi x\right),
$$

where

$$
a_{k}=\int_{-1}^{1} f(t) \cos k \pi t d t, \quad b_{k}=\int_{-1}^{1} f(t) \sin k \pi t d t .
$$

The $n$th partial sum of (1.1) will be denoted by $S_{n}(x)$. The sum

$$
S_{n}^{*}(x)=\frac{a_{0}^{*}}{2}+\sum_{k=1}^{n-1}\left(a_{k}^{*} \cos k \pi x+b_{k}^{*} \sin k \pi x\right)+\frac{a_{n}^{*}}{2} \cos n \pi x,
$$

where

$$
a_{k}^{*}=\frac{1}{n} \sum_{\alpha=-n}^{n-1} f\left(x_{\alpha}\right) \cos k \pi x_{\alpha}, \quad b_{k}^{*}=\frac{1}{n} \sum_{\alpha=-n}^{n-1} f\left(x_{\alpha}\right) \sin k \pi x_{\alpha},
$$

is called the trigonometric interpolation sum of order $n$ relative to the $2 n$ points

$$
x_{\alpha}=\frac{\alpha}{n}, \quad \alpha=-n, \cdots, 0, \cdots, n-1 .
$$

It is well known that

$$
S_{n}^{*}\left(x_{\alpha}\right)=f\left(x_{\alpha}\right), \quad \alpha=-n, \cdots, 0, \cdots, n-1,
$$

and that the partial sums of (1.3) are best approximations of $f(x)$ at the points (1.5)

Received October 13, 1969, revised December 8, 1969.

AMS Subject Classifications. Primary 4110, 4115, 4208, 6520; Secondary 4141, 4211, 6510.

Key Words and Phrases. Trigonometric approximation, interpolation series, accelerating convergence, Fourier series, Lidstone interpolation.

Copyright @ 1971, American Mathematical Society 
in the sense of least squares. It is shown by Jackson [5, pp. 22, 50, and 123] that if, for $p \geqq 1, f^{(p)}(x)$ exists and is bounded on $[-1,1]$, and if

$$
f^{(k)}(-1)=f^{(k)}(1), \quad k=0,1, \cdots, p,
$$

then there exist constants $A, B$, and $C$ such that for $n=2,3, \cdots$

$$
\begin{aligned}
\left|f(x)-S_{n}(x)\right| & \leqq(A \log n) / n^{p}, \quad-1 \leqq x \leqq 1, \\
\left|a_{n}\right| & \leqq B / n^{p}, \quad\left|b_{n}\right| \leqq B / n^{p}, \\
\left|f(x)-S_{n}^{*}(x)\right| & \leqq(C \log n) / n^{p}, \quad-1 \leqq x \leqq 1 .
\end{aligned}
$$

Although somewhat sharper results are known, for our purpose the simplified form summarized above suffices to indicate the important connection between the rate of convergence of trigonometric approximations of $f(x)$ and the smoothness and boundary conditions (1.7) satisfied by $f(x)$. Conditions (1.7) make it possible to extend the definition of $f(x)$ as a periodic function with a $p$ th derivative over the entire real line. Thus, if $f(x)$ were a smooth function on $[-1,1]$ (even analytic) but did not have the boundary behavior (1.7), its trigonometric approximations could converge extremely slowly.

Lanczos, in 1964 [8] and 1966 [9], developed a method for dealing with this problem by modifying a given function $f(x)$ by a polynomial $h_{\nu}(x)$ so that the corrected functions

$$
g_{p}(x)=f(x)-h_{p}(x)
$$

will have both the smoothness of $f(x)$ and the desired boundary behavior. He introduced a basic system of polynomials $B_{k}(x)$ that plays a central role in the method, and considered two types of modifying functions $h_{p}(x)$. We introduce a third type and show that all of the types studied so far have a simple and natural formulation in the framework of interpolation theory (Section 2). In connection with this, certain combinations of the polynomials $B_{k}(x)$ and related linear functionals are shown to form biorthogonal sets in terms of which the $h_{p}(x)$ are easily derived. We have also studied the additional question of convergence of the sequences of modifying functions $\left\{h_{p}(x)\right\}$. For each type considered we are able to give sufficient conditions for uniform convergence and uniform estimates of the truncation error (Section 4). In the course of this study, we found that the modifying function $h_{p}(x)$ of Section 2, Method 2 (considered by Lanczos the most useful) is, in fact, an interpolation polynomial introduced by Lidstone [10] in 1929 and later investigated by Whittaker [11], Widder [12], Boas and Buck [2] and others. We also found that the odd polynomials $B_{2 k+1}(x)$ coincide with the so-called Lidstone polynomials $\Lambda_{k}(x)$ and that some of Lanczos' work on this problem contributes to the study of Lidstone interpolation.

The method of accelerating convergence of trigonometric approximation is applicable to functions defined either by an analytical expression for a continuous interval or by a set of tabulated values at equally spaced points. We have earlier used the method for representing seasonal variations of ionospheric characteristics [6] and vertical profiles of ionospheric electron density [7]. As a further illustration we include here applications to the error function and its derivative (Section 2). 
2. Modifying Functions. In this section we will determine modifying functions $h_{p}(x)$ such that $g_{p}(x)$ defined by (1.9) will have a bounded $p$ th order derivative and satisfy boundary conditions of the form (1.7). Each of the cases dealt with here reduces to an interpolation problem in the following sense: Let $V$ denote a linear space of dimension $N$ over the real numbers and let $L_{1}, \cdots, L_{N}$ denote linear functionals defined on $V$. Then, given a set of real values $w_{1}, \cdots, w_{N}$, the problem of interpolation is to find an element $h$ belonging to $V$ such that

$$
L_{j}(h)=w_{i}, \quad j=1,2, \cdots, N .
$$

Elements $F_{1}, \cdots, F_{N}$ in $V$ and linear functionals $L_{1}, \cdots, L_{N}$ are said to form a biorthogonal set if

$$
\begin{aligned}
L_{j}\left(F_{k}\right)=\delta_{i k} & =0, \quad \text { if } j \neq k, \\
& =1, \text { if } j=k .
\end{aligned}
$$

It is well known [3] that a solution to (2.1) is given by

$$
h=\sum_{k=1}^{N} w_{k} F_{k},
$$

provided the $F_{k}$ and $L_{i}$ form a biorthogonal set. In the following we restrict $V$ to spaces of polynomials and consider three interpolation problems. In each case the solution is developed in the form (2.3). First, however, we must introduce Lanczos' system of polynomials.

Definition 2.1. Let the sequence $\left\{B_{k}(x)\right\}$ be defined as follows:

$$
\begin{gathered}
B_{0}(x)=1, \quad B_{1}(x)=x, \\
B_{k}^{\prime}(x)=B_{k-1}(x), \quad k=2,3, \cdots, \\
B_{2 k+1}(0)=B_{2 k+1}(1)=0, \quad k=1,2, \cdots .
\end{gathered}
$$

Remarks. The following statement can be verified by induction: For each $k=0,1,2, \cdots, B_{k}(x)$ is a uniquely determined polynomial of degree $k$ and, furthermore,

$$
\begin{aligned}
B_{2 k}(-x) & =B_{2 k}(x), & & (\text { even function) } \\
B_{2 k+1}(-x) & =-B_{2 k+1}(x), & & \text { (odd function) } \\
B_{k}^{(m)}(x) & =B_{k-m}(x), & & 0 \leqq m \leqq k \\
& =0, & & m>k .
\end{aligned}
$$

Lanczos [9] has pointed out a close connection between the $B_{k}(x)$ and Bernoulli polynomials.

The biorthogonality relations needed are established by

THEOREM 2.1. Each of the systems of functions $F_{k}$ and linear functionals $L_{i}$ given below forms a biorthogonal set:

A.

$$
\begin{aligned}
L_{i}(h) & =h^{(i)}(1)-h^{(i)}(-1), & j & =0,1, \cdots, p, \\
F_{k}(x) & =\frac{1}{2} B_{k+1}(x), & k & =0,1, \cdots, p .
\end{aligned}
$$


B.

$$
\begin{aligned}
& L_{2 j+1}(h)=h^{(2 j)}(0), \quad L_{2 j+2}(h)=h^{(2 i)}(1), \quad j=0,1, \cdots, m, \\
& F_{2 k+1}(x)=B_{2 k+1}(1-x), \quad F_{2 k+2}(x)=B_{2 k+1}(x), \quad k=0,1, \cdots, m .
\end{aligned}
$$

C.

$$
\begin{aligned}
& L_{2 j+1}(h)=h^{(2 j+1)}(0), \quad L_{2 j+2}(h)=h^{(2 j+1)}(1), \quad j=0,1, \cdots, m, \\
& F_{2 k+1}(x)=-B_{2 k+2}(1-x), \quad F_{2 k+2}(x)=B_{2 k+2}(x), \quad k=0,1, \cdots, m .
\end{aligned}
$$

Proof. In case A we have

$$
L_{i}\left(F_{k}\right)=\frac{1}{2}\left[B_{k+1}^{(j)}(1)-B_{k+1}^{(i)}(-1)\right] .
$$

Thus if $j \neq k$, then $L_{j}\left(F_{k}\right)=0$. For if $j>k+1$ then $B_{k+1}^{(j)}(x) \equiv 0$ and if $j=k+1$ then $B_{k+1}^{(i)}(x) \equiv 1$. If $j<k$ then $B_{k+1-j}(1)=B_{k+1-i}(-1)$ when $k+1-j$ is even and $B_{k+1-j}(1)=B_{k+1-j}(-1)=0$ when $k+1-j$ is odd. On the other hand, $L_{i}\left(F_{:}\right)=$ $\frac{1}{2}\left[B_{1}(1)-B_{1}(-1)\right]=1$. In case $\mathrm{B}$ we obtain the following:

$$
\begin{aligned}
L_{2 i+1}\left(F_{2 k+1}\right)=B_{2 k+1}^{(2 j)}(1) & =0, \quad \text { if } j>k, \\
& =B_{2 k+1 \ldots 2 j}(1)=0, \text { if } j<k, \\
& =1, \text { if } j=k .
\end{aligned}
$$

$$
L_{2 i+1}\left(F_{2 k+2}\right)=B_{2 k+1}^{(2 j)}(0)=0, \quad \text { if } j>k,
$$

$$
\begin{aligned}
& =B_{2 k \cdot 1-2 i}(0)=0, \text { if } j \leqq k . \\
L_{2 j+2}\left(F_{2 k+1}\right) & =B_{2 k+1}^{(2 j)}(0)=0 \quad \text { as in }(2.11) . \\
L_{2 i+2}\left(F_{2 k+2}\right) & =B_{2 k+1}^{(2)}(1)=\delta_{j k} \text { as in }(2.10) .
\end{aligned}
$$

Case $\mathrm{C}$ follows in a similar manner and hence is omitted.

We can now derive the desired modifying functions $h_{\nu}(x)$.

Method 1. Let $f(x)$ have a bounded derivative of order $p$ on $[-1,1]$. Then

$$
g_{p}(x)=f(x)-h_{p}(x)
$$

will have a bounded $p$ th order derivative on $[-1,1]$ and satisfy conditions of the form (1.7), provided $h_{p}(x)$ is a polynomial satisfying

$$
h_{p}^{(j)}(1)-h_{p}^{(j)}(-1)=f^{(j)}(1)-f^{(i)}(-1), \quad j=0,1, \cdots, p ;
$$

that is, $h_{p}(x)$ is a polynomial solution of the interpolation problem (2.1), where the functionals $L_{i}$ are defined by (2.7) and $w_{i}=f^{(i)}(1)-f^{(j)}(-1), j=0,1, \cdots, p$. By (2.3) and Theorem 2.1A, the desired $h_{p}(x)$ is given by

$$
h_{p}(x)=\frac{1}{2} \sum_{k=0}^{D}\left[f^{(k)}(1)-f^{(k)}(-1)\right] B_{k+1}(x) .
$$

The trigonometric approximations (1.1) and (1.3) will converge to $g_{p}(x)$ according to (1.8). It should be noted that if $f(x)$ is defined by a set of tabulated values, the derivatives $f^{(i)}( \pm 1)$ appearing in (2.14) must be approximated by numerical methods of differentiation (see [3] for standard techniques of numerical differentiation; Lanczos [8] discusses a special technique for approximating the derivatives of even order). In certain problems it is advantageous to have the modifying function $h_{p}(x)$ 
expressed in terms of only even- or only odd-order derivatives of $f(x)$. Such expressions are formulated by the following two methods.

Method 2. Let $f(x)$ have a bounded derivative of order $p=2 m+1$ on $[0,1]$. Define $g_{p}(x)$ as an odd function by

$$
\begin{aligned}
g_{p}(x) & =f(x)-h_{p}(x), & 0 \leqq x \leqq 1, \\
& =-g_{p}(-x), & -1 \leqq x<0,
\end{aligned}
$$

where $h_{p}(x)$ is a polynomial such that $h_{p}(0)=f(0)$, so that $g_{p}(0)=0$. Now in order for $g_{p}(x)$ to be $p$ times differentiable on $[-1,1]$, it is necessary and sufficient that

$$
g_{p}^{(i)}(0-)=g_{p}^{(i)}(0+), \quad j=0,1, \cdots, p,
$$

where $g^{(j)}(0-)$ and $g^{(j)}(0+)$ denote the left and right derivatives at $x=0$, respectively. Thus $g_{p}(x)$ must satisfy two sets of boundary conditions, $(2.16)$ and

$$
g_{p}^{(j)}(-1)=g_{p}^{(j)}(1), \quad j=0,1, \cdots, p .
$$

Although (2.16) and (2.17) comprise $4 m+4$ conditions, half of these (those with odd $j$ ) will be satisfied automatically since $g_{p}(x)$ is an odd function. For it follows from the oddness of $g_{p}(x)$ that

$$
g_{p}^{(2 k+1)}(x)=g_{p}^{(2 k+1)}(-x), \quad k=0,1, \cdots, m .
$$

Moreover, $g_{p}^{(2 k)}(x)=-g_{p}^{(2 k)}(-x)$ implies $g_{p}^{(2 k)}(0)=0$, and together with (2.17), $g_{p}^{(2 k)}(1)=0$. Therefore, it suffices that the polynomial $h_{p}(x)$ satisfy the $2 m+2$ conditions

$$
\begin{aligned}
& h_{p}^{(2 k)}(0)=f^{(2 k)}(0), \quad k=0,1, \cdots, m ; \\
& h_{p}^{(2 k)}(1)=f^{(2 k)}(1),
\end{aligned}
$$

that is, that $h_{p}(x)$ be a polynomial solution of the interpolation problem (2.1), where the functionals $L_{j}$ are given by $(2.8)$ and $w_{2 j+1}=f^{(2 i)}(0), w_{2 j+2}=f^{(2 j)}(1), j=$ $0,1, \cdots, m$. Thus, by $(2.3)$ and Theorem $2.1 \mathrm{~B}$ the desired $h_{p}(x)$ is given by

$$
h_{p}(x)=\sum_{i=0}^{m}\left[f^{(2 i)}(0) B_{2 j+1}(1-x)+f^{(2 i)}(1) B_{2 i+1}(x)\right] .
$$

This is the interpolation problem whose solution (2.20) was given by Lidstone [10]. Again we obtain a function $g_{p}(x)$ whose trigonometric approximations (1.1) and (1.3) converge according to (1.8). A useful feature of this method (pointed out by Lanczos) is that, although (2.20) involves only derivatives of $f(x)$ up to order $2 m, g_{p}(x)$ has the advantage of having a bounded derivative of order $2 m+1$. Thus, for $m=0$, no derivatives of $f(x)$ are required in (2.20), but $g_{1}(x)$ is differentiable and satisfies $g_{1}^{\prime}(-1)$ $=g_{1}^{\prime}(1)$. This case is particularly useful for approximating tabulated functions $f(x)$ whose derivatives are unknown.

Method 3. Let $f(x)$ have a bounded derivative of order $p=2 m+1$ on $[0,1]$. Let $g_{p}(x)$ be defined as an even function by

$$
\begin{array}{rlrl}
g_{p}(x) & =f(x)-h_{p}(x), & 0 \leqq x \leqq 1, \\
& =g_{p}(-x), & -1 \leqq x & <0 .
\end{array}
$$


Again we wish to determine a polynomial $h_{p}(x)$ such that $g_{p}(x)$ will be $p$ times differentiable on $[-1,1]$ and satisfy (2.17). But, as in Method 2, $g_{p}(x)$ must also satisfy (2.16), giving a total of $4 m+6$ boundary conditions. In this case more than half $(2 m+4)$ of these conditions (those with $j$ even) will be satisfied automatically since $g_{p}(x)$ is an even function and hence

$$
g_{p}^{(2 k)}(x)=g_{p}^{(2 k)}(-x), \quad k=0,1, \cdots, m+1 .
$$

Moreover, $g_{p}^{(2 k+1)}(x)=-g_{p}^{(2 k+1)}(-x)$, implies $g_{p}^{(2 k+1)}(0)=0$, and together with (2.17), $g_{p}^{(2 k+1)}(1)=0$. Thus it suffices for the polynomial $h_{p}(x)$ to satisfy the $2 m+2$ conditions

$$
\begin{aligned}
& h_{p}^{(2 k+1)}(0)=f^{(2 k+1)}(0), \quad k=0,1, \cdots, m ; \\
& h_{p}^{(2 k+1)}(1)=f^{(2 k+1)}(1),
\end{aligned}
$$

that is, that $h_{p}(x)$ be a solution to the interpolation problem (2.1), where the functionals $L_{i}$ are given by $(2.9)$ and $w_{2 j+1}=f^{(2 i+1)}(0), w_{2 j+2}=f^{(2 j+1)}(1), j=0,1$, $\cdots, m$. By (2.3) and Theorem 2.1C the desired $h_{p}(x)$ is given by

$$
h_{p}(x)=\sum_{i=0}^{m}\left[f^{(2 j+1)}(1) B_{2 i+2}(x)-f^{(2 i+1)}(0) B_{2 i+2}(1-x)\right] .
$$

All of the modifying functions (2.14), (2.20) and (2.24) arise in a simple and natural way when we consider the associated interpolation problems and biorthogonality relations of Theorem 2.1. The first two modifying functions are given by Lanczos [9]. The new function (2.24) has the advantage that the corrected function (2.21) can be extended as a periodic function with $2 m+2$ derivatives, although (2.24) involves only derivatives up to order $2 m+1$. In practice (2.24) would therefore be more appropriate than $(2.20)$ when the derivatives $f^{(i)}(0)$ and $f^{(i)}(1)$ are known up to an odd order, say, $j=2 m+1$ but not for $j=2 m+2$. For example, if the values of the first derivative $f^{\prime}(0), f^{\prime}(1)$ were given but $f^{\prime \prime}(0), f^{\prime \prime}(1)$ were unknown, then (2.24) would be more suitable than (2.20).

The following examples illustrate the use of Methods 2 and 3 in accelerating the convergence of trigonometric interpolation sums. For Method 2, $g_{p}(x)$ is odd so that its interpolation sum (1.3) reduces to a pure sine series. If this sum is truncated after $K$ terms, the resulting approximation of $f(x)$ has the form

$$
Y_{p, K}(x)=h_{p}(x)+\sum_{k=1}^{K} b_{k}^{*} \sin k \pi x, \quad 1 \leqq K \leqq n-1, \quad p=2 m+1,
$$

where

$$
b_{k}^{*}=\frac{2}{n} \sum_{\alpha=1}^{n-1} g_{p}\left(\frac{\alpha}{n}\right) \sin k \pi \frac{\alpha}{n},
$$

and where $h_{p}(x)$ and $g_{p}(x)$ are defined by (2.20) and (2.15), respectively. For Method 3, $g_{p}(x)$ is even, so that its sum (1.3) is a pure cosine series, which, when truncated after $K$ terms, gives an approproximation to $f(x)$ of the form

$$
Y_{p, K}(x)=h_{p}(x)+\frac{a_{0}^{*}}{2}+\sum_{k=1}^{K} a_{k}^{*} \cos k \pi x, \quad 1 \leqq K \leqq n-1, p=2 m+2,
$$


where

$$
a_{k}^{*}=\frac{2}{n} \sum_{\alpha=1}^{n-1} g_{p}\left(\frac{\alpha}{n}\right) \cos k \pi \frac{\alpha}{n}+\frac{1}{n}\left[g_{p}(0)+(-1)^{k} g_{p}(1)\right],
$$

and where $h_{p}(x)$ and $g_{p}(x)$ are given by (2.24) and (2.21), respectively. For convenience we list the first few polynomials $B_{k}(x)$ :

$$
\begin{array}{ll}
B_{1}(x)=x, & B_{2}(x)=\frac{x^{2}}{2}-\frac{1}{6}, \\
B_{3}(x)=\frac{x^{3}}{6}-\frac{x}{6}, & B_{4}(x)=\frac{x^{4}}{24}-\frac{x^{2}}{12}+\frac{7}{360}, \\
B_{5}(x)=\frac{x^{5}}{120}-\frac{x^{3}}{36}+\frac{7 x}{360}, & B_{6}(x)=\frac{x^{6}}{720}-\frac{x^{4}}{144}+\frac{7 x^{2}}{720}-\frac{31}{15120} .
\end{array}
$$

Example 1. Let $f(x)$ denote the error function with normalized variable

$$
f(x)=\operatorname{erf} 2 x=\frac{2}{\sqrt{ } \pi} \int_{0}^{2 x} e^{-t^{2}} d t, \quad 0 \leqq x \leqq 1 .
$$

The following values of the derivatives of $f(x)$ are used:

\begin{tabular}{cccccc}
$\nu$ & 1 & 2 & 3 & 4 & 5 \\
\hline$\sqrt{ } \pi f^{(\nu)}(0)$ & 4 & 0 & -32 & 0 & 768 \\
\hline$\sqrt{ } \pi e^{4} f^{(\nu)}(1)$ & 4 & -32 & 224 & -1280 & 4864 \\
\hline
\end{tabular}

Table 1 contains the coefficients (2.26) and (2.28), $k=0,1, \cdots, 10$, calculated with $n=200$ for each of the values $m=0,1$, and 2. Also included in Table 1 are the values of the maximum absolute error

$$
M_{p, k}=\max \left\{\left|f(\alpha / 200)-Y_{p, k}(\alpha / 200)\right|: \alpha=1,2, \cdots, 200\right\} .
$$

The tabulated values of $f(x)$ used as input were taken from [1] with ten significant digits and most of the calculations were performed with double-precision arithmetic to reduce errors of roundoff. The acceleration of convergence is evident both in the series of coefficients $a_{k}^{*}$ and $b_{k}^{*}$ and in the values of $M_{p, k}$. For example, with $p=1$, the $\left|b_{k}^{*}\right|$ decrease from $\left|b_{1}^{*}\right| \cong 3 \times 10^{-1}$ to $\left|b_{10}^{*}\right| \cong 2.10^{-5}$, whereas, with $p=5$, they decrease from $\left|b_{1}^{*}\right| \cong 4 \times 10^{-1}$ to $\left|b_{9}^{*}\right| \cong 1 \times 10^{-10}$. Also, it is seen that $M_{1,10}=7.3 \times$ $10^{-5}$, whereas $M_{5.8}=8.0 \times 10^{-10}$. In this example the convergence is somewhat faster with Method 2 than with Method 3. This is probably due to the fact that $f(x)$ is naturally an odd function. In the next example, Method 3 gives faster convergence, but then we are dealing with an even function.

Example 2. Here we consider the derivative of the error function (with normalized variable)

$$
f(x)=\frac{2}{\sqrt{\pi}} e^{-(2 x)^{2}}, \quad 0 \leqq x \leqq 1 .
$$


Table 1. Coefficients $a *{ }_{k}$ and $b *_{k}$ and Maximum Absolute Error $M_{p, k}$ From Analysis of erf $2 x, 0 \leq x \leq 1$

Method $2(\mathrm{~m}=0, \mathrm{p}=1)$

$\begin{array}{cc}\mathrm{k} & \mathrm{b}{ }_{\mathrm{k}} \\ 0 & \\ 1 & .3462463177 \\ 2 & .0259554394 \\ 3 & .0013111854 \\ 4 & -.0002449383 \\ 5 & .0001435006 \\ 6 & -.0000877068 \\ 7 & .0000570549 \\ 8 & -.0000390231 \\ 9 & .0000277945 \\ 10 & -.0000204646\end{array}$

$$
\mathrm{M}_{1, \mathrm{k}}
$$

Method $3(\mathrm{~m}=0, \mathrm{p}=2)$

$2.7 \times 10^{-2}$

$1.6 \times 10^{-3}$

$5.4 \times 10^{-4}$

$3.6 \times 10^{-4}$

2. $5 \times 10^{-4}$

1. $8 \times 10^{-4}$

$1.4 \times 10^{-4}$

$1.1 \times 10^{-4}$

8. $8 \times 10^{-5}$

7. $3 \times 10^{-5}$

Method $2(m=1, p=3)$

$$
\mathrm{k}
$$

0

$\mathrm{b} *{ }_{k}$

$$
\mathrm{M}_{3, \mathrm{k}}
$$

.3249169740

\section{.0286216074}

.0005212097

.0000883327

$-.0000271341$

.0000110401

-.0000051298

.0000026358

$-.0000014638$

.00000086468

2. $9 \times 10^{-2}$

$5.9 \times 10^{-4}$

1. $3 \times 10^{-4}$

4. $6 \times 10^{-5}$

2. $1 \times 10^{-5}$

1. $1 \times 10^{-5}$

6. $1 \times 10^{-6}$

3. $7 \times 10^{-6}$

2. $4 \times 10^{-6}$

1. $6 \times 10^{-6}$

Method $2(\mathrm{~m}=2, \mathrm{p}=5)$

$\mathrm{k}$

$$
\mathrm{b} * \mathrm{k}
$$

$$
\mathrm{M}_{5, \mathrm{k}}
$$

$\begin{array}{ll}.4113615483 & 2.7 \times 10^{-2} \\ .0259202145 & 8.8 \times 10^{-4} \\ .0008769487 & 4.4 \times 10^{-6} \\ .0000039141 & 6.2 \times 10^{-7} \\ .00000052818 & 9.2 \times 10^{-8} \\ .000000076746 & 1.6 \times 10^{-8} \\ .0000 .00013603 & 2.7 \times 10^{-9} \\ .0000000023012 & 8.0 \times 10^{-10} \\ .00000000012557 & 9.2 \times 10^{-10} \\ .0000000002 .3073 & 6.9 \times 10^{-10}\end{array}$

$$
a * k
$$

$\mathrm{M}_{2, \mathrm{k}}$

1. 4367884391

.0842190610

$4.4 \times 10^{-2}$

$-.0336517436$

9. $8 \times 10^{-3}$

$-.0065131039$

3. $3 \times 10^{-3}$

$-.0019205011$

1. $4 \times 10^{-3}$

$-.0005950163$

$7.9 \times 10^{-4}$

$-.0003421257$

4. $5 \times 10^{-4}$

$-.0001439425$

3. $1 \times 10^{-4}$

$-.0001053547$

2. $0 \times 10^{-4}$

$-.0000512496$

-.0000426522

$1.5 \times 10^{-4}$

Method $3(\mathrm{~m}=1, \mathrm{p}=4)$

$$
\mathrm{a} * \mathrm{k}
$$

$\mathrm{M}_{4, \mathrm{k}}$

\section{l. 4367884391}

.4073791302

$-.0075135659$

1. $0 \times 10^{-2}$

$-.0025234734$

2. $9 \times 19^{-3}$

$-.0002868649$

4. $0 \times 10^{-4}$

$-.0000779602$

1. $1 \times 10^{-4}$

$-.0000194321$

3. $6 \times 10^{-5}$

$-.0000093485$

1. $7 \times 10^{-5}$

$-.0000032524$

$-.0000019948$

$-.00000083105$

7. $6 \times 10^{-6}$

4. $3 \times 10^{-6}$

2. $3 \times 10^{-6}$

1. $5 \times 10^{-6}$

Method $3(\mathrm{~m}=2, \mathrm{p}=6)$

$$
\mathrm{a}{ }_{\mathrm{k}}
$$

$\mathrm{M}_{6, \mathrm{k}}$

1. 4367884391

1. 4133394885

.0049370204

$-.00] 1435552$

$-.0000923245$

$-.0000135787$

$-.0000023531$

$-.00000079797$

$-.00000021273$

$-.00000010196$

-. 000000034214
6. $0 \times 10^{-3}$

1. $3 \times 10^{-3}$

$1.1 \times 10^{-4}$

1. $7 \times 10^{-5}$

3. $5 \times 10^{-6}$

1. $2 \times 10^{-6}$

3. $9 \times 10^{-7}$

1. $8 \times 10^{-7}$

7. $4 \times 10^{-8}$

3. $910^{-8}$ 
For this example the following values of the derivatives of $f(x)$ are needed:

\begin{tabular}{cccccr}
$\nu$ & 1 & 2 & 3 & 4 & 5 \\
\hline$\sqrt{ } \pi f^{(\nu)}(0)$ & 0 & -16 & 0 & 384 & 0 \\
\hline$\sqrt{ } \pi e^{4} f^{(\nu)}(1)$ & -16 & 112 & -640 & 2432 & 1024 \\
\hline
\end{tabular}

Table 2 contains the coefficients $a_{k}^{*}$ and $b_{k}^{*}$ and values of $M_{p, k}$ calculated with $n=200$. Again it is clear that both Methods 2 and 3 accelerate the convergence, but Method 3 appears to be better suited in this example. As an illustration of this we note that $M_{3.7}=1.0 \times 10^{-4}$, whereas $M_{4.7}=3.6 \times 10^{-8}$.

3. Fourier Series for the $B_{k}(x)$. In Section 4 we shall make use of the Fourier series for the polynomials $B_{k}(x)$, which are given in the present section, together with some helpful uniform bounds. Using (1.1) and (1.2) one can easily show that the Fourier series $\beta_{1}(x)$ for $B_{1}(x)$ on $[-1,1]$ reduces to

$$
\beta_{1}(x)=2 \sum_{k=1}^{\infty}(-1)^{k+1} \frac{\sin k \pi x}{k \pi} .
$$

This series converges absolutely and uniformly to $B_{1}(x)$ on every closed subset of $(-1,1)$; at $x= \pm 1$ it converges to zero. By successive integration of $\beta_{1}(x)$, we obtain a sequence of functions $\left\{\beta_{k}(x)\right\}$ given by

$$
\begin{aligned}
& \beta_{2 n+1}(x)=2(-1)^{n+1} \sum_{k=1}^{\infty}(-1)^{k} \frac{\sin k \pi x}{(k \pi)^{2 n+1}}, \quad n=1,2, \cdots, \\
& \beta_{2 n}(x)=2(-1)^{n+1} \sum_{k=1}^{\infty}(-1)^{k} \frac{\cos k \pi x}{(k \pi)^{2 n}}, \quad n=1,2, \cdots,
\end{aligned}
$$

where the boundary conditions $\beta_{2 n+1}(0)=\beta_{2 n+1}(1)=0, n \geqq 1$, determine the constants of integration. The convergence of the series (3.2) and (3.3) is uniform and absolute for all $x$. Definition 2.1 implies that $\beta_{k}(x)=B_{k}(x)$ for $-1 \leqq x \leqq 1, k \geqq 2$.

THEOREM 3.1. For $n=1,2, \cdots$, and all $x$

$$
\left|(-1)^{n} \beta_{2 n+1}(x)-2 \sin \pi x / \pi^{2 n+1}\right| \leqq M /(2 \pi)^{2 n+1},
$$

and

$$
\left|(-1)^{n} \beta_{2 n}(x)-2 \cos \pi x / \pi^{2 n}\right| \leqq M /(2 \pi)^{2 n},
$$

where $M=8\left(\pi^{2} / 6-1\right)$.

Proof. From (3.2) and (3.3) we obtain for $n=1,2, \cdots$

$$
\left|(-1)^{n} \beta_{2 n+1}(x)-\frac{2 \sin \pi x}{(k \pi)^{2 n+1}}\right| \leqq \sum_{k=2}^{\infty} \frac{2}{(k \pi)^{2 n+1}}=\frac{2}{(2 \pi)^{2 n+1}} \sum_{k=2}^{\infty}\left(\frac{2}{k}\right)^{2 n+1},
$$

and

$$
\left|(-1)^{n+1} \beta_{2 n}(x)-\frac{2 \cos \pi x}{(2 \pi)^{2 n}}\right| \leqq \sum_{k=2}^{\infty} \frac{2}{(k \pi)^{2 n}}=\frac{2}{(2 \pi)^{2 n}} \sum_{k=2}^{\infty}\left(\frac{2}{k}\right)^{2 n} .
$$


Table 2. Coefficients $a * k$ and $b * k$ and Maximum Absolute Error $M_{p, k}$ From Analysis of $\frac{2}{\sqrt{\pi}} e^{-(2 x)^{2}}, 0 \leq x \leq 1$

Method $2(\mathrm{~m}=0, \mathrm{p}=1)$

k b*k

0

] -. 1322909918

2.1057200701

3.0306922785

4.0120668632

5.0046732464

6.0032244576

7.0015827289

8.0013239247

9.0007245222

10.0006699766

Method $2(\mathrm{~m}=1, \mathrm{p}=3)$

k b*k

$\mathrm{M}_{3, \mathrm{k}}$

0

$1-.6399096414$

3. $5 \times 10^{-2}$

2.0236045634

$1.4 \times 10^{-2}$

3.0118915881

4.0018024255

5.0006122979

6.0001831435

7.0001027920

8.0000408712

9.0000282015

$10.0000\} 30542$

2. $6 \times 10^{-3}$

9. $3 \times 10^{-4}$

3. $7 \times 10^{-4}$

2. $0 \times 10^{-4}$

1. $0 \times 10^{-4}$

$6.5 \times 10^{.5}$

$4.0 \times 10^{-5}$

2. $8 \times 10^{-5}$

Method $2(m=1 p=5)$

$\mathrm{b} * \mathrm{k}$

$\mathrm{M}_{5, \mathrm{k}}$

0

] -2.2200684770

2. $0 \times 10^{-2}$

$2 \quad-.0155101070$

3.0053888769

4.0005800920

5.0001066470

6.0000221778

.0000087741

6. $0 \times 10^{-3}$

7. $0 \times 10^{-4}$

1. $4 \times 10^{-4}$

3. $5 \times 10^{-5}$

1. $4 \times 10^{-5}$

5. $2 \times 10^{-6}$

2. $6 \times 10^{-6}$

1. $2 \times 10^{-6}$

$7.2 \times 10^{-7}$
Method $3(m=0, p=2)$

$\mathrm{a} * \mathrm{k}$

$\mathrm{M}_{2, \mathrm{k}}$

.9953222650

.5103783892

9. $3 \times 10^{-2}$

.0899174316

2. $9 \times 10^{-3}$

.0024561430

$1.0 \times 10^{-3}$

.0005550106

4. $8 \times 10^{-4}$

$-.0002131106$

2. $7 \times 10^{-4}$

.0001040505

1. $6 \times 10^{-4}$

$-.0000564048$

$1.1 \times 10^{-4}$

.0000331222

7. $3 \times 10^{-5}$

$-.0000206943$

.0000135823

5. $3 \times 10^{-5}$

3. $9 \times 10^{-5}$

Method $3(\mathrm{~m}=1, \mathrm{p}=4)$

$\mathrm{a} * \mathrm{k}$

$\mathrm{M}_{4, \mathrm{k}}$

.9953222650

.6461652090

.0814307553

$8.6 \times 10^{-2}$

.0041325235

$4.2 \times 10^{-3}$

.0000245933

2. $8 \times 10^{-5}$

.0000041483

$5.0 \times 10^{-6}$

$-.00000072329$

.00000014953

$-.000000028876$

$8.9 \times 10^{-7}$

$1.8 \times 10^{-7}$

3. $6 \times 10^{-8}$

1. $8 \times 10^{-8}$

.0000000017781

2. $0 \times 10^{-8}$

.0000000036552

$1.6 \times 10^{-8}$

Method $3(\mathrm{~m}=2, \mathrm{p}=6)$

$$
\mathrm{a} * \mathrm{k}
$$

$M_{6, k}$

.9953222650

.6681781391

.0810868033

.0041627196

.0000192190

.0000055572

-.0000011951

.00000033664

$8.5 \times 10^{-2}$

4. $2 \times 10^{-3}$

2. $4 \times 10^{-5}$

7. $3 \times 10^{-6}$

1. $7 \times 10^{-6}$

5. $3 \times 10^{-7}$

$1.9 \times 10^{-7}$

-.00000011285

.000000043199

8. $0 \times 10^{-8}$

$-.000000018358$
3. $6 \times 10^{-8}$

1. $8 \times 10^{-8}$ 
It suffices to set

$$
M=2 \sum_{k=2}^{\infty}\left(\frac{2}{k}\right)^{2}=8\left(\frac{\pi^{2}}{6}-1\right), \quad n \geqq 2 .
$$

A proof of (3.4) was given in [12].

THEOREM 3.2. For $n=0,1,2, \cdots$, and all $x$

$$
\left|\beta_{n}(x)\right| \leqq M_{1} / \pi^{n}
$$

where $M_{1}=2\left(\pi^{2} / 3-1\right)$.

Proof. From Theorem 3.1

$$
\left|\beta_{2 n+1}(x)\right| \leqq\left(2+M / 2^{2 n+1}\right) / \pi^{2 n+1}, \quad n=1,2, \cdots,
$$

and

$$
\left|\beta_{2 n}(x)\right| \leqq\left(2+M / 2^{2 n}\right) / \pi^{2 n}, \quad n=1,2, \cdots,
$$

where $M=8\left(\pi^{2} / 6-1\right)$. Thus it suffices to let $M_{1}=2+M / 2$. The cases $n=0,1$ follow by inspection.

4. Convergence of Interpolation Series. Three interpolation problems were solved in Section 2 by the polynomials $h_{p}(x)$ defined by $(2.14),(2.20)$, and (2.24). Taking the limit as $p \rightarrow \infty$, we obtain interpolation series. In this section we give sufficient conditions for uniform convergence of these series and explicit error bounds for the partial sums. Our results will be expressed in terms of functions of class $Q$.

Definition 4.1. A function $f(x)$ is said to belong to class $Q$ if $f(x)$ is entire and if there exists a positive number $q<\pi$ such that

$$
f^{(m)}(0)=O\left(q^{m}\right), \quad(m \rightarrow \infty) .
$$

Remarks. Let $\rho(f)$ denote the order of an entire function $f(z)$ of a complex variable $z$ and let $\tau(f)$ denote the type of its order. Then it can be shown that class $Q$ consists of all entire functions $f(z)$ such that either $[0 \leqq \rho(f)<1]$ or $[\rho(f)=1$ and $0 \leqq \tau(f)<\pi$ ]. As examples of functions in class $Q$ we mention all polynomials and the transcendental functions $e^{a z}, \sin a z$ and $\cos a z$ with $|a|<\pi$. The following useful property of class $Q$ will be needed, a proof of which is given in [12]:

LEMMA 4.1. Let $f(x)$ belong to class $Q$ and let $[a, b]$ be an arbitrary closed finite interval. Then there exists a positive number $q<\pi$ such that

$$
f^{(m)}(x)=O\left(q^{m}\right) \quad(m \rightarrow \infty)
$$

uniformly on $a \leqq x \leqq b$.

Widder [12], in 1942, proved the following theorem for Lidstone series, which arises from (2.20).

THEOREM 4.2. Let $f(x)$ belong to class $Q$. Then the series

$$
f(0) B_{1}(1-x)+f(1) B_{1}(x)+f^{(2)}(0) B_{3}(1-x)+f^{(2)}(1) B_{3}(x)+\cdots
$$

converges uniformly to $f(x)$ on $[0,1]$. Moreover, there exists a constant $M_{2}$ and $a$ positive number $q<\pi$ such that

$$
\left|f(x)-f_{n}(x)\right| \leqq M_{2}\left(\frac{q}{\pi}\right)^{n}, \quad 0 \leqq x \leqq 1, n=1,2, \cdots,
$$

where $f_{n}(x)$ denotes the nth partial sum of (4.3). 
We shall now derive similar results for the interpolation series arising from (2.14) and (2.24). For that purpose we shall make use of the following two theorems.

THEOREM 4.3. If $f^{(m+1)}(x)$ exists and is continuous on $[-1,1]$, then for $-1 \leqq x \leqq 1$

$$
\begin{aligned}
f(x)= & \frac{1}{2} \int_{-1}^{1} f(t) d t+\frac{1}{2} \sum_{k=0}^{m}\left[f^{(k)}(1)-f^{(k)}(-1)\right] B_{k+1}(x) \\
& -\frac{1}{2} \int_{-1}^{1} f^{(m+1)}(t) \beta_{m+1}(x-t-1) d t .
\end{aligned}
$$

Remark. Theorem 4.3 was derived by Lanczos [9, Eq. (16.38)] and hence will not be proved here. The following is a similar result.

THEOREM 4.4. Let $f^{(2 m+1)}(x)$ exist and be continuous on $[0,1]$. Then

$$
f(x)=H_{m}(x)+G_{m}(x), \quad 0 \leqq x \leqq 1,
$$

where

$$
H_{m}(x)=\int_{0}^{1} f(t) d t+\sum_{k=1}^{m}\left[f^{(2 k-1)}(1) B_{2 k}(x)-f^{(2 k-1)}(0) B_{2 k}(1-x)\right]
$$

and

$$
G_{m}(x)=\frac{1}{2} \int_{0}^{1} f^{(2 m+1)}(t)\left[\beta_{2 m+1}(x+t-1)-\beta_{2 m+1}(x-t-1)\right] d t .
$$

Proof. From successive integration by parts we obtain, for $0 \leqq x \leqq 1$,

$$
\begin{aligned}
2 G_{0}(x) & =\int_{0}^{1} f^{\prime}(t)\left[\beta_{1}(x+t-1)-\beta_{1}(x-t-1)\right] d t \\
& =\sum_{k=1}^{2 m}\left\{f^{(k)}(t)\left[(-1)^{k-1} \beta_{k+1}(x+t-1)+\beta_{k+1}(x-t-1)\right]\right\}_{t=0}^{1}+2 G_{m}(x) .
\end{aligned}
$$

Now using the periodicity property $\beta_{n}(x+2)=\beta_{n}(x)$ and cancelling terms in the above sum, we have

$$
G_{0}(x)=\sum_{k=1}^{m}\left[f^{(2 k-1)}(1) B_{2 k}(x)-f^{(2 k-1)}(0) B_{2 k}(1-x)\right]+G_{m}(x) .
$$

Here we have also replaced $\beta_{2 k}(x)$ by $B_{2 k}(x)$ and $\beta_{2 k}(x-1)$ by $B_{2 k}(x-1)=B_{2 k}(1-x)$. On the other hand we have, for $0 \leqq x \leqq 1$,

$$
\begin{aligned}
2 G_{0}(x) & =\int_{0}^{1} f^{\prime}(t) \beta_{1}(x+t-1) d t-\int_{0}^{x} f^{\prime}(t) \beta_{1}(x-t-1) d t-\int_{x}^{1} f^{\prime}(t) \beta_{1}(x-t-1) d t \\
& =\int_{0}^{1} f^{\prime}(t)(x+t-1) d t-\int_{0}^{x} f^{\prime}(t)(x-t-1) d t-\int_{x}^{1} f^{\prime}(t)(x-t+1) d t \\
& =2\left[\int_{\theta}^{1} t f^{\prime}(t) d t+f(x)-f(1)\right] \\
& =2\left[f(x)-\int_{0}^{1} f(t) d t\right] .
\end{aligned}
$$

Combining (4.9) and (4.10) gives (4.6) and this completes the proof. 
We come now to the two main theorems of this section.

THEOREM 4.5. If $f(x)$ belongs to class $Q$, then the series

$$
\frac{1}{2} \int_{-1}^{1} f(t) d t+\frac{1}{2} \sum_{k=0}^{\infty}\left[f^{(k)}(1)-f^{(k)}(-1)\right] B_{k+1}(x)
$$

converges uniformly to $f(x)$ on $-1 \leqq x \leqq 1$. If $f_{n}(x)$ denotes the nth partial sum of (4.11) then there exists a positive number $q<\pi$ such that

$$
\left|f(x)-f_{n}(x)\right| \leqq M\left(\frac{q}{\pi}\right)^{n}, \quad-1 \leqq x \leqq 1, \quad n=1,2,3, \cdots .
$$

for some constant $M$ independent of $n$.

Proof. By Theorem 4.3

$$
\left|f(x)-f_{n}(x)\right|=\frac{1}{2}\left|\int_{-1}^{1} f^{(n+1)}(t) \beta_{n+1}(x-t-1) d t\right| .
$$

By Lemma 4.1 there exist positive numbers $K$ and $q<\pi$ such that

$$
\left|f^{n+1}(t)\right| \leqq K q^{n+1}, \quad-1 \leqq t \leqq 1, \quad n=1,2, \cdots .
$$

Combining this with the bound on $\beta_{n+1}(x-t-1)$ given by Theorem 3.2, we obtain

$$
\left|f(x)-f_{n}(x)\right| \leqq\left(K M_{1} q\right)\left(\frac{q}{\pi}\right)^{n}, \quad-1 \leqq x \leqq 1, \quad n=1,2, \cdots
$$

This completes the proof.

THEOREM 4.6. If $f(x)$ belongs to class $Q$, then the series

$$
\int_{0}^{1} f(t) d t+f^{(1)}(1) B_{2}(x)-f^{(1)}(0) B_{2}(1-x)+f^{(3)}(1) B_{4}(x)+f^{(3)}(0) B_{4}(1-x)+\cdots
$$

converges uniformly to $f(x)$ on $0 \leqq x \leqq 1$. If $f_{n}(x)$ denotes the nth partial sum of (4.13), then there exists a positive constant $q<\pi$ such that

$$
\left|f(x)-f_{n}(x)\right| \leqq M\left(\frac{q}{\pi}\right)^{n}, \quad 0 \leqq x \leqq 1, \quad n=1,2, \cdots
$$

for some constant $M$ independent of $n$.

Proof. By Theorem 4.4

$$
\left|f(x)-f_{2 m+1}(x)\right|=\left|f(x)-H_{m}(x)\right|=\left|G_{m}(x)\right|,
$$

where $H_{m}(x)$ and $G_{m}(x)$ are given by (4.7) and (4.8), respectively. But by Lemma 4.1 . there exist positive numbers $K$ and $q<\pi$ such that

$$
\left|f^{(n)}(t)\right| \leqq K q^{n}, \quad 0 \leqq t \leqq 1, \quad n=1,2, \cdots .
$$

Combining this with the bounds for $\beta_{2 m+1}(x)$ asserted by Theorem 3.2, we obtain

$$
\left|f(x)-f_{2 m+1}(x)\right| \leqq K M_{1}\left(\frac{q}{\pi}\right)^{2 m+1} .
$$


Thus

$$
\begin{aligned}
\left|f(x)-f_{2 m+2}(x)\right| & =\left|f(x)-f_{2 m+1}(x)-f^{(2 m+1)}(1) B_{2 m+2}(x)\right| \\
& \leqq\left|f(x)-f_{2 m+1}(x)\right|+\left|f^{(2 m+1)}(1) B_{2 m+2}(x)\right| \\
& \leqq \frac{2 \pi K M_{1}}{q}\left(\frac{q}{\pi}\right)^{2 m+2},
\end{aligned}
$$

where, in deriving the last inequality, we have used (4.15), (4.16) and (3.6). The proof follows immediately.

Widder [12] proved that a real function can be expanded in an absolutely convergent Lidstone series (4.3) if and only if it is the difference of two minimal completely convex functions. Widder's proof made strong use of the estimates (3.4) and the additional bound

$$
(-1)^{2 n+1} \beta_{2 n+1}(x) \geqq M_{3} / \pi^{2 n}, \quad n=1,2, \cdots .
$$

Since bounds of the form (4.17) do not exist for the even functions $\beta_{2 n}(x)$, it seems unlikely that a similar characterization can be obtained for expansions of the form (4.13).

ESSA Research Laboratories

Boulder, Colorado 80302

1. M. AbRamowitz \& I. Stegun, Handbook of Mathematical Functions, with Formulas, Graphs and Mathematical Tables, Nat. Bur. Standards Appl. Math. Series, 55, Superintendent of Documents, U.S. Government Printing Office, Washington, D.C., 1964, chap. 7. MR 29 \#4914.

2. R. P. Boas, JR. \& R. C. Buck, Polynomial Expansions of Analytic Functions, 2nd rev. ed., Ergebnisse der Mathematik und ihrer Grenzgebiete, Band 19, Academic Press, New York and Springer-Verlag, Berlin and New York, 1964. MR 29 \#218.

3. PHILIP J. DAvis, Interpolation and Approximation, Blaisdell, Waltham, Mass., 1963, pp. 28-30. MR 28 \#393.

4. E. IsAacson \& H. B. Keller, Analysis of Numerical Methods, Wiley, New York, 1966. MR 34 \#924.

5. D. JACKson, The Theory of Approximation, Amer. Math. Soc. Colloq. Publ., vol. XI, Amer. Math. Soc., Providence, R.I., 1930.

6. WILliaM B. JONES \& G. HARDY, Numerical Prediction of Ionospheric Characteristics, ESSA Technical Report ERL 76-ITS 66, U.S. Government Printing Office, Washington, D.C., 1968.

7. William B. Jones \& F. STewart, Numerical Mapping of Ionospheric Plasma Frequency, paper presented at URSI Spring Meeting in Washington, D.C., 1969.

8. C. Lanczos, "Evaluation of noisy data,"J. Soc. Indust. Appl. Math. Ser. B Numer. Anal., v. 1, 1964, pp. 76-85. MR 31 \#4146.

9. C. Lanczos, Discourse on Fourier Series, Hafner, New York, 1966. MR 33 \#7772.

10. G. J. LIDSTONE, "Notes on the extension of Aitken's theorem (for polynomial interpolation) to the Everett types," Proc. Edinburgh Math. Soc. (2), v. 2, 1929, pp. 16-19.

11. J. M. WHITTAKER, "On Lidstone series and two-point expansions of analytic functions," Proc. London Math. Soc., v. 36, 1934, pp. 451-469.

12. D. V. WIDDER, "Completely convex functions and Lidstone series," Trans. Amer. Math. Soc., v. 51, 1942, pp. 387-398. MR 3, 293. 